\title{
Decreased microRNA-768-3p expression indicates a poor prognosis in patients with breast cancer and promotes breast cancer cell viability, migration and invasion
}

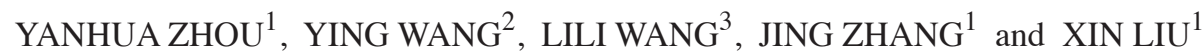 \\ Departments of ${ }^{1}$ Oncology, ${ }^{2}$ Quality Control Division and ${ }^{3}$ Operating Room, \\ Affiliated Hospital of Weifang Medical University, Weifang, Shandong 261031, P.R. China
}

Received November 6, 2020; Accepted February 24, 2021

DOI: $10.3892 / \mathrm{ol} .2021 .12840$

\begin{abstract}
Breast cancer is the most common malignancy in women and microRNA-768-3p (miR-768-3p) is abnormally expressed in hepatocellular carcinoma, non-small cell lung carcinomas and melanoma. The aim of the present study was to evaluate the prognostic value and biological function of miR-768-3p in breast cancer. The expression of miR-768-3p in tumor tissues and adjacent tissues of 116 patients with breast cancer obtained by surgery and normal breast cell lines MCF-10A and breast cancer cell lines (MCF-7, MDA-MB-231, T-47D and SK-BR-3) were detected by reverse transcription-quantitative PCR. The association between miR-768-3p expression and the clinicopathological characteristics of patients was analyzed using the $\chi^{2}$ test. In addition, the Kaplan-Meier method was used for survival analysis. A Cox regression model was used to examine the effect of miR-768-3p on the prognosis of patients with breast cancer. Hemocytometer cell counting and Transwell assays were used to detect the effects of miR-768-3p on the characteristics of breast cancer cells. The target genes of miR-768-3p in breast cancer were identified by bioinformatics software and detected by luciferase reporter assay. Compared with normal tissues and normal breast cancer cells, miR-768-3p was significantly decreased in breast cancer tissues and cancer cells $(\mathrm{P}<0.001)$. The reduction in miR-768-3p was significantly associated with lymph node metastasis $(\mathrm{P}=0.040)$, Tumor Node Metastasis stage $(\mathrm{P}=0.035)$, and cancer subtype $(\mathrm{P}=0.008)$. In addition, patients with low miR-768-3p expression had a shorter overall survival time (log-rank $\mathrm{P}=0.022$ ) compared with those with high expression and miR-768-3p may be a potential prognostic marker (hazard ratio $=4.637 ; 95 \%$ confidence
\end{abstract}

Correspondence to: Dr Yanhua Zhou, Department of Oncology, Affiliated Hospital of Weifang Medical University, 2428 Yuhe Road, Weifang, Shandong 261031, P.R. China

E-mail: yanhua_zhou858@163.com

Key words: microRNA-768-3p, prognosis, progression, breast cancer interval=1.296-16.597; $\mathrm{P}=0.018)$. When transfected with miR-768-3p inhibitor, cell viability, migration and invasion were significantly promoted compared with the control group $(\mathrm{P}<0.05)$. In addition, eukaryotic translation initiation factor 4E (eIF4E) was the target gene of miR-768-3p in breast cancer. All experiments confirmed that miR-768-3p, a tumor suppressor, inhibited the viability, migration and invasion of breast cancer cells through eIF4E. miR-768-3p may be a potential prognostic marker of breast cancer and may participate in the progression of breast cancer.

\section{Introduction}

Breast cancer is a malignant tumor derived from breast tissue (1). Breast cancer is one of the leading causes of cancer-related death in women and the second most common type of cancer in the world (2). There were 266,120 newly diagnosed cases and 40,920 deaths from breast cancer in the USA in 2018 (2). Although great progress has been made in the treatment of breast cancer in the past decade, the 5-year survival rate of some patients is only $24 \%$ (3). The increase in tumor incidence and tumor-specific death is almost always caused by recurrence and metastasis (4). Hence, finding viable breast cancer prognostic markers and potential treatment targets is necessary for the treatment of patients with breast cancer.

MicroRNAs (miRNAs) are small, highly conserved non-coding RNAs consisting of 21-25 nucleotides that serve an important role in RNA silencing and posttranscriptional regulation of gene expression (5). Previous studies have confirmed that dysregulated miRNAs are involved in the progression of various cancers, including breast cancer $(6,7)$. In addition, studies in recent years have found that abnormally expressed miRNAs in tissues and serum can be used as biomarkers for tumor prognosis and diagnosis, for example, miR-519a was downregulated in gastric cancer and was associated with poor prognosis (8). The downregulation of serum miR-218-5p is a biomarker for prostatic bone metastasis (9).

miR-768-3p is a highly expressed miRNA whose role has been described in a hepatitis B virus-related hepatocellular carcinoma and hurthle cell carcinoma $(10,11)$. miR-768-3p is abnormally expressed in non-small cell lung cancer tissues 
and cell lines A549 and HCC4006, and is involved in tumor viability, migration and invasion (12). Compared with the expression level in gastric cancer tissues, the expression level of miR-768-3p in non-tumor tissues is higher (13). Zheng et al (14) performed a study with 77 breast cancer and 17 control tissues from frozen mammoplasty samples in 2016 and found 34 differentially expressed miRNAs, including miR-768-3p in the downregulated miRNAs. However, the clinical and biological role of miR-768-3p in breast cancer remains unclear.

The aim of the present study was to investigate the expression and clinical association of miR-768-3p in breast cancer and to explore the regulatory effect of miR-768-3p on breast cancer cell function. The finding of the present study indicated that miR-768-3p may be a new prognostic breast cancer marker and a potential target for the molecular treatment of breast cancer.

\section{Materials and methods}

Patients and tissue specimens. Surgically resected tumor tissues and adjacent tissues which were obtained $5 \mathrm{~cm}$ away from breast cancer tissues of 116 patients with breast cancer were collected from the Affiliated Hospital of Weifang Medical University (Weifang, China) from June 2011 to June 2014. All patients' tissue specimens were confirmed to be breast cancer and the patients did not receive any chemotherapy, adjuvant therapy, or immunotherapy before surgery. Exclusion criteria for the patients were: i) Multiple cancers or cancers of other organs; ii) previous axillary surgery; or iii) history of benign breast diseases. Patients were followed for 5 years and the days from surgery to the last follow-up or death were recorded at every 3 months for the first 2 years after surgery, then once every 6 months (between 2-4 years) and thereafter once yearly (after 4 years) by telephone to analyze the effects of miRNA changes on overall survival. The present study was approved by the Research Ethics Committee of Affiliated Hospital of Weifang Medical University (Weifang, China; approval no. WYFY20110609001). All participants in the study signed written informed consent. Patient sample tissues were processed and anonymized according to ethical and legal standards. The research methodology followed the standards of the Helsinki Declaration. The clinicopathological features of the patients (age range, 32-89 years; median age, 60 years) are listed in Table I. TNM stage analysis of the patients was done according to the 2010 American Joint Committee on Cancer recommendation for Tumor-lymph Node Metastasis Classification (AJCC 7th edition) (15).

Cell lines and transfection. MCF-10A, a normal breast cell line and breast cancer cell lines MCF-7 and MDA-MB-231 were purchased from the Chinese Academy of Science Cell Bank (Shanghai, China). The breast cancer cell lines T-47D and SK-BR-3 were obtained from American Type Culture Collection (ATCC). MCF-10A and all breast cancer cells were cultured in DMEM (Thermo Fisher Scientific Inc.) containing $10 \%$ fetal bovine serum (FBS, Invitrogen; Thermo Fisher Scientific Inc.) and 1\% penicillin/streptomycin (Gibco; Thermo Fisher Scientific Inc.) in a humidified incubator with $5 \% \mathrm{CO}_{2}$ at $37^{\circ} \mathrm{C}$. The miR-768-3p mimic (50 nM), mimic negative control (NC, $50 \mathrm{nM})$, miR-768-3p inhibitor $(50 \mathrm{nM})$ and inhibitor $\mathrm{NC}(50 \mathrm{nM})$ were purchased from Guangzhou RiboBio Co., Ltd. for in vitro regulation of breast cancer cells. Untransfected cells were the control group. The sequences of used were as follows: miR-768-3p mimic, 5'-UCACAAUGC UGACACUCAAACUGCUGAC-3'; miR-768-3p inhibitor, 5'-GUCAGCAGUUUGAGUGUCAGCAUUGUGA-3' mimic NC, 5'-UUCUCCGAACGUGUCACGUTTACGUGACAC GUUCGGAGAATT-3' and inhibitor NC, 5'-CAGUAC UUUUGUGUAGUACAA-3'. The transfection reagent was Lipofectamine $2000^{\circledR}$ (Invitrogen; Thermo Fisher Scientific Inc.). According to the requirements of the transfection reagent, transfection was conducted in an incubator at $37^{\circ} \mathrm{C}$ for $6 \mathrm{~h}$ and subsequently the medium was replaced with fresh DMEM medium. Follow-up experiments were performed $48 \mathrm{~h}$ after transfection.

Reverse transcription-quantitative $(R T-q) P C R$. TRIzol ${ }^{\circledR}$ (Invitrogen; Thermo Fisher Scientific Inc.) reagent was added to patient tissues and cells lines (MCF-10A, SK-BR-3, MCF-7, T-47D and MDA-MB-231) according to the manufacturer's instructions. miRNA was purified from tissues and cells using a miRNA Purification kit (CoWin Biosciences). The extracted total RNA was then reverse transcribed into cDNA using the miRNA cDNA Synthesis kit (CoWin Biosciences) according to the manufacturer's protocol. qPCR was performed on a 7300 real-time PCR system using a SYBR Green miRNA qPCR Assay kit (CoWin Biosciences). The thermocycling condition used were as follows: $95^{\circ} \mathrm{C}$ for $10 \mathrm{~min}$ followed by 40 cycles of $94^{\circ} \mathrm{C}$ for $15 \mathrm{sec}$, $55^{\circ} \mathrm{C}$ for $30 \mathrm{sec}$ and $70^{\circ} \mathrm{C}$ for $30 \mathrm{sec}$. The relative expression of miR-768-3p was calculated by the $2^{-\triangle \Delta C q}$ method (16), and U6 was used as the reference gene for mRNA quantification. The following primer sequences were used: miR-768-3p forward 5'-GCCGAGUCACAAUGCUGACACUCA-3' and reverse 5'-CTCGTTCGGCAGCACA-3'; and U6 forward 5'-AACGCT TCACGAATTTGCGT-3' and reverse 5'-CTCGTTCGGCAG CACA-3'. Each sample was tested in triplicate.

Cell viability assay. Breast cancer cells MCF-7 and MDA-MB-231 transfected with the miR-768-3p mimic and miR-768-3p inhibitor and corresponding NCs were seeded in 96-well plates at a density of $1 \times 10^{4}$ cells/well. The cells were dissociated into a single cells suspensions with $0.05 \%$ trypsin (Invitrogen; Thermo Fisher Scientific Inc.) at $24 \mathrm{~h}$ intervals, and then washed once with PBS. Finally, the cells were counted manually using a hemocytometer and observed under a light microscope (magnification, x100). Experiments were repeated in triplicate.

Cell migration and invasion assay. Transwell assays were used to detect changes in cell migration and invasion. Transfected MCF-7 and MDA-MB-231 cells were prepared as a single cell suspension in serum-free medium and $8 \times 10^{4}$ cells were added to the upper chamber of the Matrigel-coated Transwell chamber $\left(37^{\circ} \mathrm{C}\right.$ for $3 \mathrm{~h}$ to form the Matrigel layer in the chamber) or the upper chamber of the Matrigel-free chamber for determination of the invasion or migration ability, respectively. DMEM medium $(500 \mu \mathrm{l})$ containing $10 \% \mathrm{FBS}$ was added to the lower chamber of the Transwell chamber. After $24 \mathrm{~h}$ of culture in the incubator at $37^{\circ} \mathrm{C}$, the non-migrated and non-invaded cells in the upper chamber were wiped clean with cotton swabs, fixed 
Table I. Association between miR-768-3p expression levels and clinicopathological features in patients with breast cancer ( $\mathrm{n}=116)$.

miR-768-3p expression

\begin{tabular}{|c|c|c|c|c|}
\hline & & & & \\
\hline Parameters & No of cases & Low $(n=65)$ & High $(n=51)$ & P-value \\
\hline Age, years & & & & 0.350 \\
\hline$\leq 50$ & 61 & 37 & 24 & \\
\hline$>50$ & 55 & 28 & 27 & \\
\hline Tumor size, cm & & & & 0.460 \\
\hline$\leq 2$ & 59 & 31 & 28 & \\
\hline$>2$ & 57 & 34 & 23 & \\
\hline Differentiation & & & & 0.095 \\
\hline Well-defined + Moderate & 60 & 29 & 31 & \\
\hline Poor & 56 & 36 & 20 & \\
\hline Lymph node metastasis & & & & 0.040 \\
\hline Negative & 62 & 29 & 33 & \\
\hline Positive & 54 & 36 & 18 & \\
\hline TNM stage & & & & 0.035 \\
\hline I-II & 71 & 34 & 37 & \\
\hline III-IV & 45 & 31 & 14 & \\
\hline Subtypes & & & & 0.008 \\
\hline Luminal A & 52 & 21 & 31 & \\
\hline Luminal B & 22 & 13 & 9 & \\
\hline HER-2 upregulation & 14 & 9 & 5 & \\
\hline Triple-negative breast cancer & 28 & 22 & 6 & \\
\hline
\end{tabular}

TNM, tumor node metastasis; HER2, human epidermal growth factor 2; miR, microRNA.

with $4 \%$ paraformaldehyde for $10 \mathrm{~min}$ at room temperature and stained with $0.1 \%$ crystal violet for $10 \mathrm{~min}$ at room temperature. Then the chamber was flushed with water until the water was no longer purple. Five fields were randomly selected under an light microscope (magnification, x200) for counting. Changes in cell migration and invasion were manually measured.

Luciferase reporter assay. Bioinformatics TargetScan v.7.2 was used to (Whitehead Institute for Biomedical Research, Massachusetts Institute of Technology) predict the target genes of miR-768-3p. Then the dual-luciferase reporter assay (Promega Corporation) was used to verify the results. The sequence Wild-type (WT) and mutant (Mut) 3'UTR of eukaryotic translation initiation factor 4E (eIF4E) was synthesized by Sangon Biotech, Co., Ltd, and then inserted into the luciferase reporter pmiRGLO vector (Promega Corporation). MCF-7 cells were seeded in 24-well plates containing DMEM at a density of $5 \times 10^{4}$ and cultured overnight in an incubator at $37^{\circ} \mathrm{C}$. WT or Mut PmiRglo-3'-UTR-eIF4E, renin luciferase plasmid and miR-768-3p mimic or inhibitor (Guangzhou RiboBio Co., Ltd.) were transfected into cells. The transfection reagent was Lipofectamine $2000^{\circledR}$ (Invitrogen; Thermo Fisher Scientific Inc.). The sequences used were as follows: miR-768-3p mimic, 5'-UCACAAUGCUGACACUCAAACUGCUGAC-3'; miR-768-3p inhibitor, 5'-GUCAGCAGUUUGAGUGUCAGC AUUGUGA-3' mimic NC, 5'-UUCUCCGAACGUGUCACG UTTACGUGACACGUUCGGAGAATT-3' and inhibitor NC,
5'-CAGUACUUUUGUGUAGUACAA-3'. After $48 \mathrm{~h}$, the MCF-7 cells were collected and $200 \mu 1$ reporter lysis buffer (Promega Corporation) was added. Then, the luciferase activity was determined by the Luciferase Assay System (Promega Corporation). Renilla luciferase activity was used for normalization.

Statistical analysis. Statistical analysis of the present study was performed using GraphPad Prism 7.0 software (GraphPad Software Inc.) and SPSS 22.0 software (IBM Corp.) and all data are presented as the mean \pm SD. All experiments were repeated 3 times. The difference between the breast cancer tissues and normal adjacent tissues was detected by a paired Student's t-test. Comparisons between multiple groups were performed using one-way ANOVA followed by the post hoc Tukey's test. A $\chi^{2}$ test was used to evaluate the association between $\mathrm{miR}-768-3 \mathrm{p}$ expression and the clinicopathological features of patients with breast cancer. Kaplan-Meier and log-rank tests were used for survival analyses. Cox regression analysis was used to evaluate the effect of miR-768-3p on the prognosis of patients with breast cancer. $\mathrm{P}<0.05$ was considered to indicate a statistically significant difference.

\section{Results}

Expression of miR-768-3p in breast cancer tissues and cell lines. To detect the expression pattern of miR-768-3p in breast cancer tissues, RT-qPCR was performed with 116 normal 
A

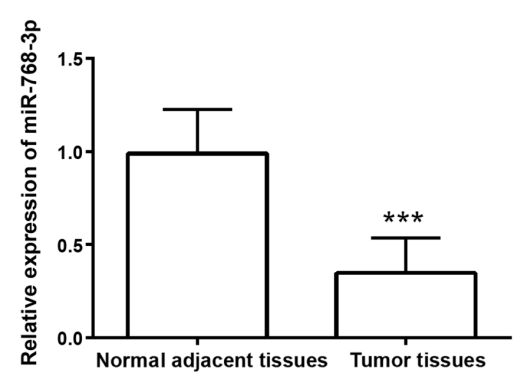

B

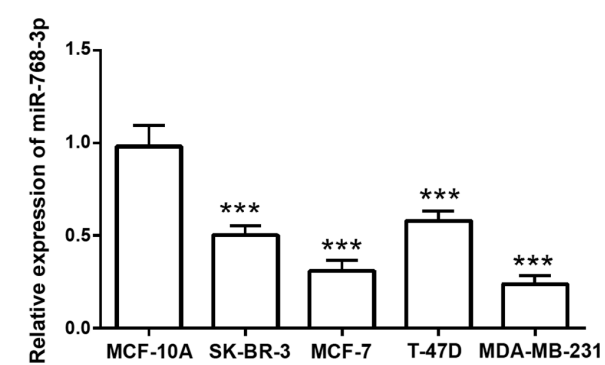

Figure 1. Expression levels of miR-768-3p in breast cancer tissues and cells detected by RT-qPCR. (A) Expression levels of miR-768-3p in tumor and normal adjacent tissues of patients with breast cancer $(n=116)$. The expression of miR-768-3p was significantly lower in breast cancer tissues compared with that in adjacent normal tissues. (B) Expression of miR-768-3p in breast cancer cells (SK-BR3, MCF-7, T-47D and MDA-MB-231) and normal breast cells (MCF-10A). Compared with normal cells, breast cancer cells had significantly decreased miR-768-3p. ${ }^{* * *} \mathrm{P}<0.001$. miR, microRNA; RT-q, reverse transcription-quantitative.

adjacent and tumor tissues. Compared with normal adjacent tissues, cancer tissues had significantly decreased miR-768-3p expression $(\mathrm{P}<0.001$; Fig. 1A). In addition, compared with normal MCF-10A breast cells, breast cancer cells had a significantly decreased expression level of miR-768-3p $(\mathrm{P}<0.001$; Fig. 1B), which was consistent with the expression results in tissues. As the MCF-7 and MDA-MB-231 had the lowest expression of miR-768-3p of the breast cancer cells tested, they were used for subsequent experimentation. The aforementioned results suggested that miR-768-3p is a tumor suppressor miRNA in breast cancer.

miR-768-3p expression is associated with the clinicopathological characteristics of patients with breast cancer. Association between the expression level of miR-768-3p and the clinicopathological characteristics of patients with breast cancer was further explored. According to the average expression level of miR-768-3p $(0.346 \pm 0.189)$ in the tissue samples of the patients as the cutoff value, the breast cancer patients were divided into the high miR-768-3p expression group $(n=51)$ and the low miR-768-3p expression group $(n=65)$. A $\chi^{2}$ test was used to analyze the relationship between miR-768-3p and the clinicopathological features of the patients and the results are shown in Table I. Low expression of miR-768-3p was significantly associated with lymph node metastasis, tumor node metastasis (TNM) stage and cancer subtype $(\mathrm{P}<0.05$; Table I), but not with age, tumor size or differentiation ( $\mathrm{P}>0.05$; Table I). The aforementioned findings indicated that the reduction of miR-768-3p expression may serve a crucial role in the breast cancer.

Low expression of miR-768-3p is associated with poor prognosis in breast cancer. According to the 5-year follow-up information of patients with breast cancer, the Kaplan-Meier method and log-rank test were used to examine the relationship between miR-768-3p expression and the survival time of patients with breast cancer and to explore the prognostic value of miR-768-3p in patients with breast cancer. The survival curve is shown in Fig. 2. Patients with low expression of miR-768-3p had a shorter overall survival time (log-rank $\mathrm{P}=0.022$; Fig. 2) compared with patients with high expression of miR-768-3p. In addition, multivariate Cox regression was used to analyze the effect of miR-768-3p on the prognosis of patients with breast cancer and the results are shown in

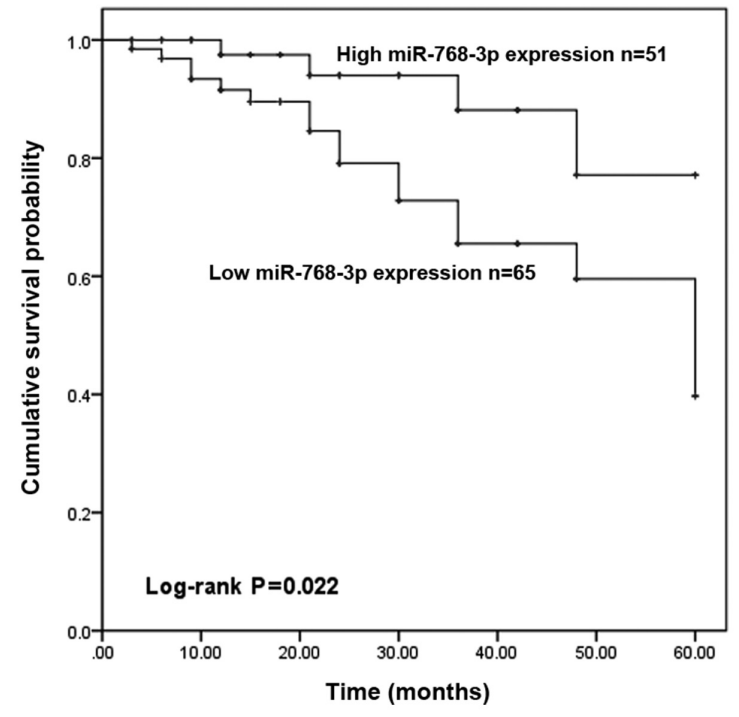

Figure 2. Kaplan-Meier analysis of the effects of miR-768-3p expression on overall survival time. Patients with breast cancer with low expression of miR-768-3p had a shorter overall survival time compared with those with high expression (log-rank $\mathrm{P}=0.022$ ). $\mathrm{miR}$, microRNA.

Table II. miR-768-3p [hazard ratio $(\mathrm{HR})=4.637$; 95\% confidence interval $(\mathrm{CI})=1.296-16.597 ; \mathrm{P}=0.018]$, lymph node metastasis $(\mathrm{HR}=0.111 ; 95 \% \mathrm{CI}=0.013-0.935 ; \mathrm{P}=0.043)$, TNM stage $(\mathrm{HR}=2.756 ; 95 \% \mathrm{CI}=1.063-7.144 ; \mathrm{P}=0.037)$, subtypes $(\mathrm{HR}=2.789 ; 95 \% \mathrm{CI}=1.055-7.376 ; \mathrm{P}=0.039$; Table II] can all be used as independent prognostic factor for patients with breast cancer. In summary, the results confirmed that low expression of miR-768-3p is associated with poor prognosis of breast cancer.

miR-768-3p inhibits cell viability, migration, and invasion in vitro. To explore the role of $\mathrm{miR}-768-3 \mathrm{p}$ in breast cancer, this study also examined the effects of miR-768-3p on breast cancer cell viability, migration, and invasion. miR-768-3p mimic and miR-768-3p inhibitor and their corresponding NCs were transfected into MCF-7 and MDA-MB-231 breast cancer cells. The changes in miR-768-3p expression in the cells were detected by RT-qPCR. The expression level of the miR-768-3p mimic group was significantly higher compared with that in the control and mimic NC groups, while that of the 
A

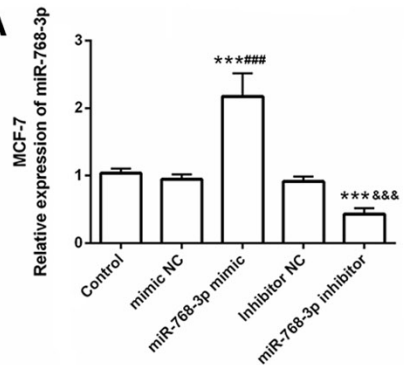

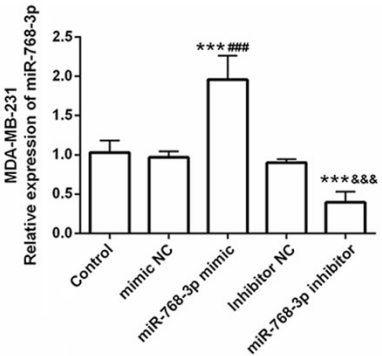

B
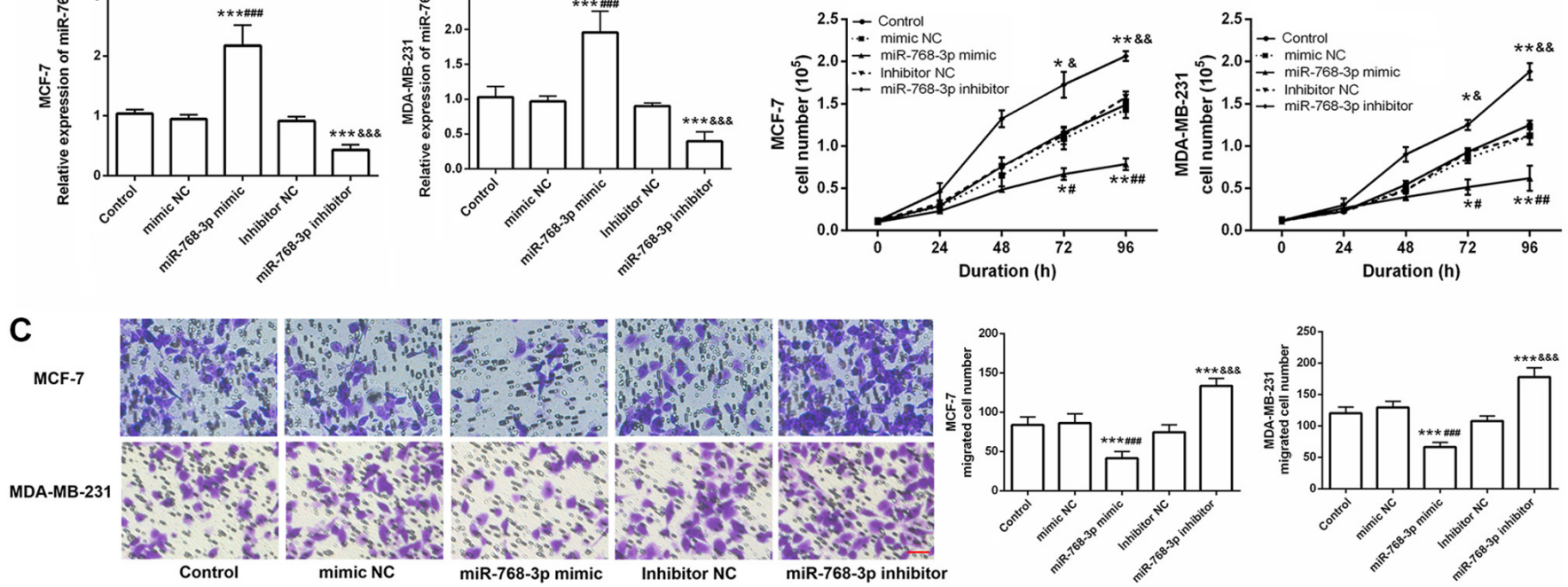

\section{D \\ MCF-7}
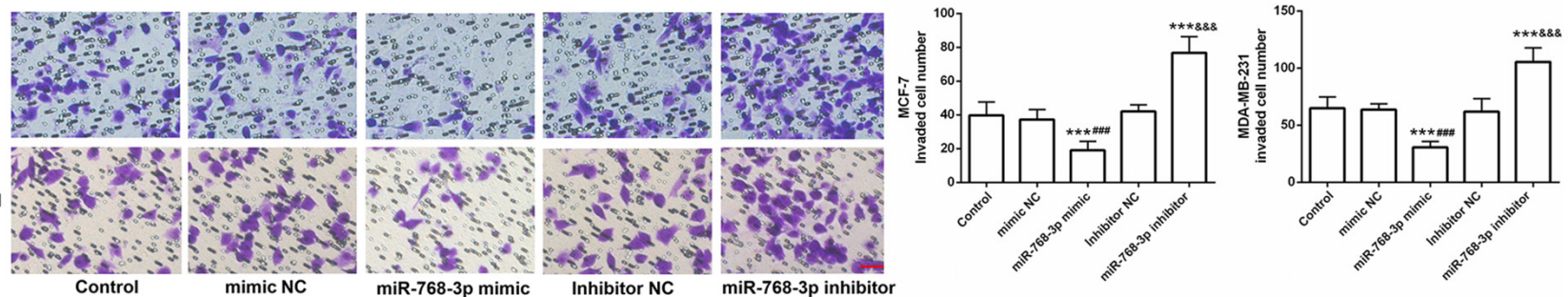

Figure 3. Effects of miR-768-3p on the function of breast cancer cells in vitro. (A) Expression level of miR-768-3p in cells after transfection with the miR-768-3p mimic or inhibitor and corresponding controls using RT-qPCR. The results confirmed that the miR-768-3p mimic and inhibitor had a higher transfection efficiency in cancer cells compared with the control, mimic NC and inhibitor NC groups, respectively $\left({ }^{* * *} \mathrm{P}<0.001\right.$, compared with control; ${ }^{\# \# \# ~} \mathrm{P}<0.001$, compared with mimic $\mathrm{NC}$; \&\&\& $\mathrm{P}<0.001$ compared with inhibitor NC). (B) Viability ability was examined. Compared with the control group, the miR-768-3p mimic group significantly inhibited cell viability, while the miR-768-3p inhibitor group significantly promoted cell viability $\left({ }^{*} \mathrm{P}<0.05\right.$, ${ }^{* *} \mathrm{P}<0.01$, compared with control; ${ }^{\#} \mathrm{P}<0.05,{ }^{\# \#} \mathrm{P}<0.001$, compared with mimic NC; ${ }^{\circledR} \mathrm{P}<0.05$, \& ${ }^{\&} \mathrm{P}<0.001$, compared with inhibitor NC). (C) Representative images and quantitative analysis of cell migration by transwell assays. (D) Representative images and quantitative analysis of cell invasion by transwell assays. Compared with the control group, the miR-768-3p mimic group significantly promoted cell migration and invasion, while the miR-768-3p inhibitor group significantly promoted cell migration and invasion. The randomly chosen fields were photographed (magnification, $\mathrm{x} 200){ }^{* * * *} \mathrm{P}<0.001$, compared with control; ${ }^{\# \# \#} \mathrm{P}<0.001$, compared with mimic NC; \&\&\&P<0.001, compared with inhibitor NC) (scale bar=200 $\mu \mathrm{m}$ ). miR, microRNA; RT-q, reverse transcription-quantitative; NC, negative control; control, untransfected cells.

Table II. Multivariate Cox analysis of miR-768-3p and clinical parameters in relation to overall survival.

\begin{tabular}{lccc}
\hline & \multicolumn{3}{c}{ Multivariate analysis } \\
\cline { 2 - 4 } Features & HR & 95\% CI & P-value \\
\hline miR-768-3p expression & 4.637 & $1.296-16.597$ & 0.018 \\
Age & 0.398 & $0.133-1.196$ & 0.101 \\
Tumor size & 0.531 & $0.188-1.500$ & 0.232 \\
Differentiation & 0.424 & $0.125-1.443$ & 0.170 \\
Lymph node metastasis & 0.111 & $0.013-0.935$ & 0.043 \\
TNM stage & 2.756 & $1.063-7.144$ & 0.037 \\
Subtypes & 2.789 & $1.055-7.376$ & 0.039 \\
\hline
\end{tabular}

TNM, tumor node metastasis; HR, hazard ratio; CI, confidence interval; miR, microRNA.

miR-768-3p inhibitor group was significantly lower compared with the control and inhibitor NC groups $(\mathrm{P}<0.001$; Fig. $3 \mathrm{~A})$. The experimental results confirmed the high transfection efficiency of the miR-768-3p mimic and miR-768-3p inhibitor in breast cancer cells.

A hemocytometer was used to detect the effect of miR-768-3p on cell viability. Compared with mimic NC or inhibitor NC, overexpression of miR-768-3p significantly inhibited cell viability in both MCF-7 and MDA-MB-231 cells, while knockdown of miR-768-3p significantly promoted cell viability $(\mathrm{P}<0.05$; Fig. $3 \mathrm{~A})$. In addition, a Transwell assay was used to analyze the effects of miR-768-3p expression on cell migration and invasion abilities of breast cancer cells. Compared with those of the control and mimic NC groups, the cell migration and invasion abilities of the miR-768-3p mimic group were inhibited, while cell migration and invasion abilities of the miR-768-3p inhibitor group were enhanced in both cell lines compared with the control and inhibitor $\mathrm{NC}$ groups $(\mathrm{P}<0.05$; Fig. $3 \mathrm{C}$ and $\mathrm{D})$.

miR-768-3p targeted eIF4E in breast cancer cells. Finally, in order to understand the molecular mechanism of miR-768-3p in breast cancer cells, bioinformatics software was used to search for the target genes of miR-768-3p and it was found that eukaryotic translation initiation factor $4 \mathrm{E}$ (eIF4E) was the target gene of miR-768-3p (Fig. 4A). Luciferase reporter 
A

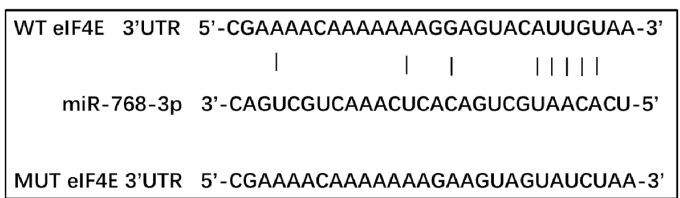

B

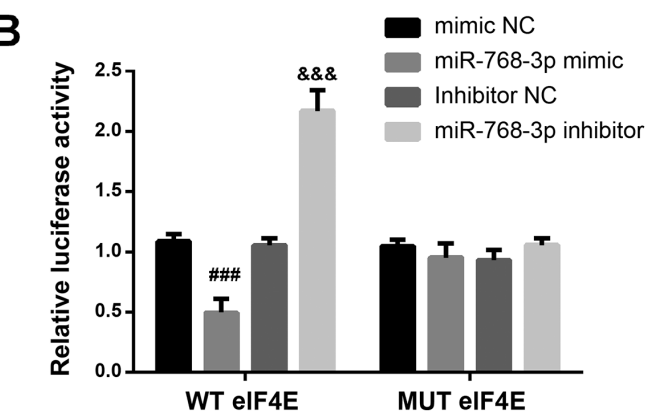

Figure 4. eIF4E was the target of miR-768-3p in breast cancer. (A) Schematic diagram of binding sites between eIF4E mRNA 3'UTR sequence and miR-768-3p was performed using TargetScan v.7.2. The binding sites were linked by short vertical lines. (B) Luciferase reporter assay was used to

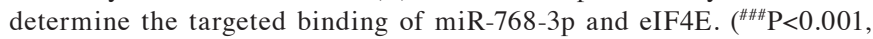
compared with mimic $\mathrm{NC}$; \&\&\& $\mathrm{P}<0.001$, compared with inhibitor $\mathrm{NC}$ ). $\mathrm{miR}$, microRNA; RT-q, reverse transcription-quantitative; NC, negative control; WT, wild-type; Mut, mutant; EIF4E, eukaryotic translation initiation factor 4E; control, untransfected cells.

assay demonstrated that luciferase activity was significantly inhibited or increased when co-transfected with wild-type eIF4E and miR-768-3p mimic or inhibitor compared with co-transfection of those with mutant eIF4E (Fig. 4B). The results indicated that miR-768-3p may affect the biological function of breast cancer cells by targeting eIF4E.

\section{Discussion}

Cancer is one of the most common life-threatening diseases in the world and breast cancer commonly occurs in women (17). Although significant advances have been made in the diagnosis and treatment of breast cancer in recent years, there were 2.1 million new breast cancer cases worldwide in 2018, so the prevention and treatment of breast cancer remains a concern and challenge for oncologists worldwide $(18,19)$. For the early detection of breast cancer, surgical treatment, chemotherapy and adjuvant therapy can significantly improve survival, but for patients with metastasis and recurrence, the identification of potentially valuable prognostic markers is critical for the treatment of breast cancer (20).

Numerous previous studies have confirmed that miRNAs are involved in a series of complex processes of tumor regulation, including tumor viability, migration, invasion and apoptosis (21-23). For example, miR-937 was upregulated in breast cancer and regulated the viability and apoptosis of breast cancer cells MCF-7 by targeting apoptotic protease activating factor 1 (24). Recent studies have found that abnormally expressed miRNAs in tumors can be widely used as a biomarker for tumor diagnosis and prognosis (25-27). For example, elevated miR-19b expression can be used as a prognostic marker for breast cancer and was involved in tumor progression through the PI3K/AKT pathway (28). The upregulation of miR-206 and the downregulation of miR-145 were related to the poor prognosis of patients with breast cancer and were important indicators to predict the prognosis of patients with breast cancer (29).

miR-768-3p has been shown to be abnormally expressed in a variety of cancers. For example. Zheng et al (14) found 34 differentially expressed miRNAs in 77 breast cancer and 17 control samples from frozen mammoplasty samples in a 2016 study, and miR-768-3p was among the downregulated miRNAs. The expression level of miR-768-3p in thyroid cancer was downregulated and the difference in miR-768-3p expression between cancerous and benign thyroid tissue was $>5$ fold (30). Similarly, miR-768-3p was downregulated in melanoma and promoted the survival and viability of melanoma cells by increasing the expression of target gene elF4E (31).

In the present study, the expression level of miR-768-3p in patients with breast cancer was assessed. The results demonstrated that the expression of miR-768-3p was decreased in cancer tissues compared with normal adjacent tissues of patients with breast cancer, which was consistent with the results of a 2016 study by Zheng et al (14). In addition, in the present study, miR-768-3p expression was also decreased in 4 breast cancer cell lines compared with normal MCF-10A cells. The findings of the present study indicated that miR-768-3p acts as a tumor suppressor miRNA in breast cancer. To test this hypothesis, the present study explored the relationship between miR-768-3p expression and the clinicopathological characteristics of patients with breast cancer and the results demonstrated that low expression of miR-768-3p was significantly associated with lymph node metastasis, TNM stage and breast cancer subtype. Hence, miR-768-3p may be involved in the development of this malignant tumor as a tumor suppressor miRNA in patients with breast cancer. In addition, survival analysis in the present study demonstrated that the overall survival time of patients with low expression of miR-768-3p was shorter compared with that of patients with high expression, suggesting that low expression of miR-768-3p was associated with poor overall survival in patients with breast cancer. Finally, the multivariate Cox regression model performed in the present study confirmed that miR-768-3p was an independent prognostic factor in patients with breast cancer. To the best of our knowledge, the present study is the first study to examine the clinical significance of miR-768-3p in patients with breast cancer and to confirm that miR-768-3p can serve as a biomarker for breast cancer prognosis.

In addition to the clinical application of miR-768-3p in breast cancer, the effect of miR-768-3p on breast cancer cell function in vitro was also assessed in the present study. A study has demonstrated that inhibiting miR-768-3p significantly reduced the viability, migration and invasion ability in non-small cell lung cancer (12). In the present study, it was first confirmed that the miR-768-3p mimic and inhibitor were successfully transfected into breast cancer cells. Viability and transwell assays performed in the present study demonstrated that compared with their respective control groups, overexpression of miR-768-3p could significantly inhibit cell viability, migration and invasion, while downregulation of miR-768-3p could significantly promote cell viability, migration and invasion. The experimental results of the present study confirmed that miR-768-3p, as a tumor suppressor miRNA, was involved in the occurrence and development of breast cancer. A previous 
study had confirmed that miR-768-3p serves an important role in inhibiting eIF4E expression and mRNA response and also regulates melanoma viability and survival in vitro (31). eIF4E recognizes and bind to the 5'cap structure of mRNA and delivers it to the eIF4E complex to initiate translation (32). The interaction between glycerol phosphate dehydrogenase and eIF4E promotes the development of pancreatic cancer (33). Astrocyte-elevated gene-1 induced gastric cancer metastasis by upregulation of eIF4E expression (34). In addition, eIF4E can be used as a therapeutic target for ovarian cancer, prostate cancer, lung cancer and other cancers, and the eIF4E-directed therapies LY2275796 (anti-sense oligonucleotides directed against eIF4E) and ribavirin (which reduces eIF4E-dependent translation) are already in clinical trials (35-39).

Hence, it was hypothesized that the regulatory effect of miR-768-3p in breast cancer may be realized through eIF4E. In the present study, it was confirmed by the luciferase reporter assay that eIF4E was indeed the target gene of miR-768-3p. The findings of the present study suggested that miR-768-3p may affect the biological functions of breast cancer cells, such as viability, migration and invasion by targeting eIF4E. It should be noted that the present study had certain limitations. Firstly, there was no significant association found between tumor differentiation and prognosis in the present study, which may be due to the small sample size. Therefore, a future study with a large sample is needed for verification of the findings of the present study. Secondly, the role of miR-768-3p in the regulation of breast cancer was not verified in in vivo animal tumor model experiments. Future studies should investigate the specific mechanism of miR-768-3p in regulating breast cancer using in vivo experiments.

In summary, the present study confirmed through a series of experiments that miR-768-3p is a tumor suppressor in breast cancer. In the present study, miR-768-3p was downregulated in breast cancer and promoted cell viability, migration, and invasion though eIF4E. Finally, in the present study the low expression of miR-768-3p was significantly related to the poor prognosis of patients with breast cancer and may be a potential prognostic marker for breast cancer.

\section{Acknowledgements}

The authors would like to thank Dr Yunxia Liu (Department of Internal Medicine, Fuyanshan Branch of Affiliated Hospital of Weifang Medical University) for her contribution to the data analysis of this study.

\section{Funding}

No funding was received.

\section{Availability of data and materials}

The datasets used and/or analyzed during the current study are available from the corresponding author on reasonable request.

\section{Authors' contributions}

YZ, YW, LW, JZ and XL contributed to the study conception and design. YZ, LW and JZ were involved in material preparation, performed the experiments and data collection and analysis. XL supplied critical reagents. YW revised the manuscript for important intellectual content. All the authors wrote the manuscript. YZ and LW confirm the authenticity of all the raw data. All the authors have read and approved the final manuscript.

\section{Ethics approval and consent to participate}

This study was approved by the Research Ethics Committee of Affiliated Hospital of Weifang Medical University (Weifang, China; approval no. WYFY20110609001). All participants in the study signed written informed consent. The research methodology meets the standards set out in the Declaration of Helsinki.

\section{Patient consent for publication}

Not applicable.

\section{Competing interests}

The authors declare that they have no competing interests.

\section{References}

1. Guo R, Chen Y, Borgard H, Jijiwa M, Nasu M, He M and Deng Y: The function and mechanism of lipid molecules and their roles in the diagnosis and prognosis of breast cancer. Molecules 25: 4864, 2020.

2. Gu Y, Wu G, Zou X, Huang P and Yi L: Prognostic value of site-specific metastases and surgery in de novo stage IV triple-negative breast cancer: A population-based analysis. Med Sci Monit 26: e920432, 2020.

3. Wang M, Ji S, Shao G, Zhang J, Zhao K, Wang Z and Wu A: Effect of exosome biomarkers for diagnosis and prognosis of breast cancer patients. Clin Transl Oncol 20: 906-911, 2018.

4. Li RH, Chen M, Liu J, Shao CC, Guo CP, Wei XL, Li YC, Huang WH and Zhang GJ: Long noncoding RNA ATB promotes the epithelial-mesenchymal transition by upregulating the miR-200c/Twist1 axe and predicts poor prognosis in breast cancer. Cell Death Dis 9: 1171, 2018.

5. Su M, Niu Y, Dang Q, Qu J, Zhu D, Tang Z and Gou D: Circulating microRNA profiles based on direct S-Poly(T)Plus assay for detection of coronary heart disease. J Cell Mol Med 24: 5984-5997, 2020.

6. Xiao Y, Humphries B, Yang C and Wang Z: miR-205 dysregulations in breast cancer: The Complexity and Opportunities. Noncoding RNA 5: 53, 2019.

7. Chong ZX, Yeap SK and Ho WY: Dysregulation of miR-638 in the progression of cancers. Pathol Res Pract: Jan 29, 2021 (Online ahead of print).

8. Cai H, Lin H, Cao W, Sun J,Huang Y and Fang Y: Downregulation of miR-519a predicts poor prognosis and contributes to tumor progression in gastric cancer. Oncol Res Treat 43: 19-26, 2020.

9. Peng P, Chen T, Wang Q, Zhang Y, Zheng F, Huang S, Tang Y, Yang C, Ding W, Ren D, et al: Decreased miR-218-5p levels as a serum biomarker in bone metastasis of prostate cancer. Oncol Res Treat 42: 165-185, 2019.

10. Zhou J, Yu L, Gao X, Hu J, Wang J, Dai Z, Wang JF, Zhang Z Lu S, Huang X, et al: Plasma microRNA panel to diagnose hepatitis B virus-related hepatocellular carcinoma. J Clin Oncol 29: 4781-4788, 2011.

11. Petric R, Gazic B, Goricar K, Dolzan V, Dzodic R and Besic N: Expression of miRNA and occurrence of distant metastases in patients with hurthle cell carcinoma. Int J Endocrinol 2016: 8945247, 2016.

12. Xie Z, Chen W, Chen Y, Wang X, Gao W and Liu Y: miR-768-3p is involved in the viability, invasion and migration of non-small cell lung carcinomas. Int J Oncol 51: 1574-1582, 2017.

13. Guo J, Miao Y, Xiao B, Huan R, Jiang Z, Meng D and Wang Y: Differential expression of microRNA species in human gastric cancer versus non-tumorous tissues. J Gastroenterol Hepatol 24: 652-657, 2009. 
14. Zheng T, Zhang X, Wang Y and Yu X: Predicting associations between microRNAs and target genes in breast cancer by bioinformatics analyses. Oncol Lett 12: 1067-1073, 2016.

15. Edge SB and Compton CC: The American Joint Committee on Cancer: The 7th edition of the AJCC cancer staging manual and the future of TNM. Ann Surg Oncol 17: 1471-1474, 2010.

16. Livak KJ and Schmittgen TD: Analysis of relative gene expression data using real-time quantitative PCR and the 2(-Delta Delta C(T)) method. Methods 25: 402-408, 2001.

17. Zhang P, Fan C, Du J, Mo X and Zhao Q: Association of miR-1247-5p expression with clinicopathological parameters and prognosis in breast cancer. Int J Exp Pathol 99: 199-205, 2018.

18. Fahad Ullah M: Breast cancer: Current perspectives on the disease status. Adv Exp Med Biol 1152: 51-64, 2019.

19. Berry DA, Cronin KA, Plevritis SK, Fryback DG, Clarke L, Zelen M, Mandelblatt JS, Yakovlev AY, Habbema JD and Feuer EJ; Cancer Intervention and Surveillance Modeling Network (CISNET) Collaborators: Effect of screening and adjuvant therapy on mortality from breast cancer. N Engl J Med 353: 1784-1792, 2005.

20. Foulkes WD, Smith IE and Reis-Filho JS: Triple-negative breast cancer. N Engl J Med 363: 1938-1948, 2010.

21. Zhou W, Gong J, Chen Y, Chen J, Zhuang Q, Cao J, Mei Z and $\mathrm{Hu}$ B: Long noncoding RNA LINC00899 suppresses breast cancer progression by inhibiting miR-425. Aging (Albany NY) 11: 10144-10153, 2019.

22. Rahman MM, Brane AC and Tollefsbol TO: MicroRNAs and epigenetics strategies to reverse breast cancer. Cells 8: 1214, 2019.

23. Weng YS, Tseng HY, Chen YA, Shen PC, Al Haq AT, Chen LM, Tung YC and Hsu HL: MCT-1/miR-34a/IL-6/IL-6R signaling axis promotes EMT progression, cancer stemness and M2 macrophage polarization in triple-negative breast cancer. Mol Cancer 18: 42, 2019.

24. Fang H, Jiang W, Jing Z, Mu X and Xiong Z: miR-937 regulates the viability and apoptosis via targeting APAF1 in breast cancer. Onco Targets Ther 12: 5687-5699, 2019.

25. Li F: Expression of miR-221 and miR-489 in breast cancer patients and their relationship with prognosis. Oncol Lett 19: $1523-1529,2020$

26. Sun R, Muheremu A and Hu Y: miRNA-30c can be used as a target in the diagnosis and treatment of osteosarcoma. Onco Targets Ther 11: 9091-9099, 2018

27. Wang F, Dai M, Chen H, Li Y, Zhang J, Zou Z and Yang H: Prognostic value of hsa-mir-299 and hsa-mir-7706 in hepatocellular carcinoma. Oncol Lett 16: 815-820, 2018.
28. Li C, Zhang J, Ma Z, Zhang F and Yu W: miR-19b serves as a prognostic biomarker of breast cancer and promotes tumor progression through $\mathrm{PI} 3 \mathrm{~K} / \mathrm{AKT}$ signaling pathway. Onco Targets Ther 11: 4087-4095, 2018.

29. Quan Y, Huang X and Quan X: Expression of miRNA-206 and miRNA-145 in breast cancer and correlation with prognosis. Oncol Lett 16: 6638-6642, 2018.

30. Vriens MR, Weng J, Suh I, Huynh N, Guerrero MA, Shen WT, Duh QY, Clark OH and Kebebew E: MicroRNA expression profiling is a potential diagnostic tool for thyroid cancer. Cancer 118: 3426-3432, 2012.

31. Jiang CC, Croft A, Tseng HY, Guo ST, Jin L, Hersey P and Zhang XD: Repression of microRNA-768-3p by MEK/ERK signalling contributes to enhanced mRNA translation in human melanoma. Oncogene 33: 2577-2588, 2014.

32. Pisera A, Campo A and Campo S: Structure and functions of the translation initiation factor eIF4E and its role in cancer development and treatment. J Genet Genomics 45: 13-24, 2018.

33. Ma X, Li B, Liu J, Fu Y and Luo Y: Phosphoglycerate dehydrogenase promotes pancreatic cancer development by interacting with eIF4A1 and eIF4E. J Exp Clin Cancer Res 38: 66, 2019.

34. Wu S, Yang L, Wu D, Gao Z, Li P, Huang W and Wang X: AEG-1 induces gastric cancer metastasis by upregulation of eIF4E expression. J Cell Mol Med 21: 3481-3493, 2017.

35. Jin J, Xiang W, Wu S, Wang M, Xiao M and Deng A: Targeting eIF4E signaling with ribavirin as a sensitizing strategy for ovarian cancer. Biochem Biophys Res Commun 510: 580-586, 2019.

36. Zhao Y, Yan M, Yun Y, Zhang J, Zhang R, Li Y, Wu X, Liu Q, Miao W and Jiang H: MicroRNA-455-3p functions as a tumor suppressor by targeting eIF4E in prostate cancer. Oncol Rep 37: 2449-2458, 2017.

37. Liu F, Wang X, Li J, Gu K, Lv L, Zhang S, Che D, Cao J, Jin S and $\mathrm{Yu}$ Y: miR-34c-3p functions as a tumour suppressor by inhibiting eIF4E expression in non-small cell lung cancer. Cell Prolif 48: 582-592, 2015

38. Karaki S, Andrieu C, Ziouziou H and Rocchi P: The eukaryotic translation initiation Factor 4E (eIF4E) as a therapeutic target for cancer. Adv Protein Chem Struct Biol 101: 1-26, 2015.

39. Sobocan M, Smolle MA, Schatz C and Haybaeck J: The interplay of tumor stroma and translational factors in endometrial cancer. Cancers (Basel) 12: 2074, 2020.

This work is licensed under a Creative Commons Attribution-NonCommercial-NoDerivatives 4.0 International (CC BY-NC-ND 4.0) License. 